Rev. Elev. Méd. vét. Pays trop., 1976, 29 (3) : 221-226.

\title{
Note sur le parasitisme dû aux nématodes et aux coccidies chez les espèces domestiques dans la région de Kaedi (Mauritanie)
}

\author{
par J. CABARET (*)
}

\begin{abstract}
RÉSUMÉ
Une enquête parasitaire a été réalisée sur les principales espèces domestiques de la région de Kaedi, de septembre 1974 à avril 1975. Une grille Strongles-Strongyloïdes-Coccidies pour les ruminants et Strongles-Ascaris pour les asins recouvre l'essentiel du parasitisme.

Les taux d'infestation sont élevés et sont susceptibles de variations saisonnières chez les ruminants. Les traitements de masse en septembre-octobre peuvent avoir un intérêt au plan sanitaire pour les Strongles et les Strongyloỉdes s'ils sont effectués sur un maximum d'animaux dans une région donnée et sur les principales espèces domestiques. L'intérêt économique de telles mesures demande des expérimentations complémentaires qui tiendraient compte de toutes les variables mises en jeu.
\end{abstract}

Les opérations relatées dans cet article se sont déroulées de juillet 1974 à mars 1975 dans la région de Kaedi, en République Islamique de Mauritanie. Les travaux sont regroupés en deux rubriques : enquête parasitaire-expérimentations.

\section{ENQUÊTE PARASITAIRE}

\section{A. Matériel et méthodes}

\section{a) Matériel}

L'origine géographique des prélèvements est :

- clinique de l'Inspection régionale de l'Élevage de Kaedi : 327 ;

- ville de Kaedi et environs proches : 419 ;

- autre : 429.

Les résultats sont représentatifs de la région définie par les localités suivantes: Ould Yengé, M'bout, Aleg, Boghé.

(") 151 bis, rue Jean-Jaurès, 94700 Maisons-Alfort, France.
Les examens coprologiques sont réalisés sur les principales espèces domestiques. Un intérêt particulier est porté aux petits ruminants étant donné :

- leur importance économique dans la région ;

- le lourd tribut qu'ils payent au parasitisme ;

- la facilité de leur contention pour l'obtention de prélèvements et l'administration de vermifuges.

\section{b) Méthodes}

L'analyse coprologique est réalisée sur 2 à $5 \mathrm{~g}$ de fèces prélevées dans le rectum. Le prélèvement est enrichi par la contrifugation et éclairci à l'antiformine. L'examen microscopique porte sur des lamelles $18 \mathrm{~mm} \times 18 \mathrm{~mm}$. Le nombre d'éléments parasitaires compté sur la préparation, multiplié par un facteur 100 , correspond approximativement à la teneur par gramme de fèces.

Des analyses réalisées en faible nombre en 
début de l'enquête ont permis de dégager les grands traits du parasitisme accessible à ce type d'analyse et aboutit à la grille : StrongleStrongylö̈des-Coccidie.

Des examens de viscères ont eu lieu à l'abattoir de Kaedi dans le but de préciser l'incidence de l'osophagostomose bovine.

\section{B. Résultats}

Ils sont précisés par les graphiques.

Les strongles dominants des ruminants sont des Haemonchus. Les Trichostrongylus et Cooperia sont bien représentés. En fin de saison des pluies, les diverses espèces de strongles sont présentes. Durant la saison sèche, on assiste à une réduction des espèces. Les coccidies les plus fréquentes appartiennent au genre Eimeria
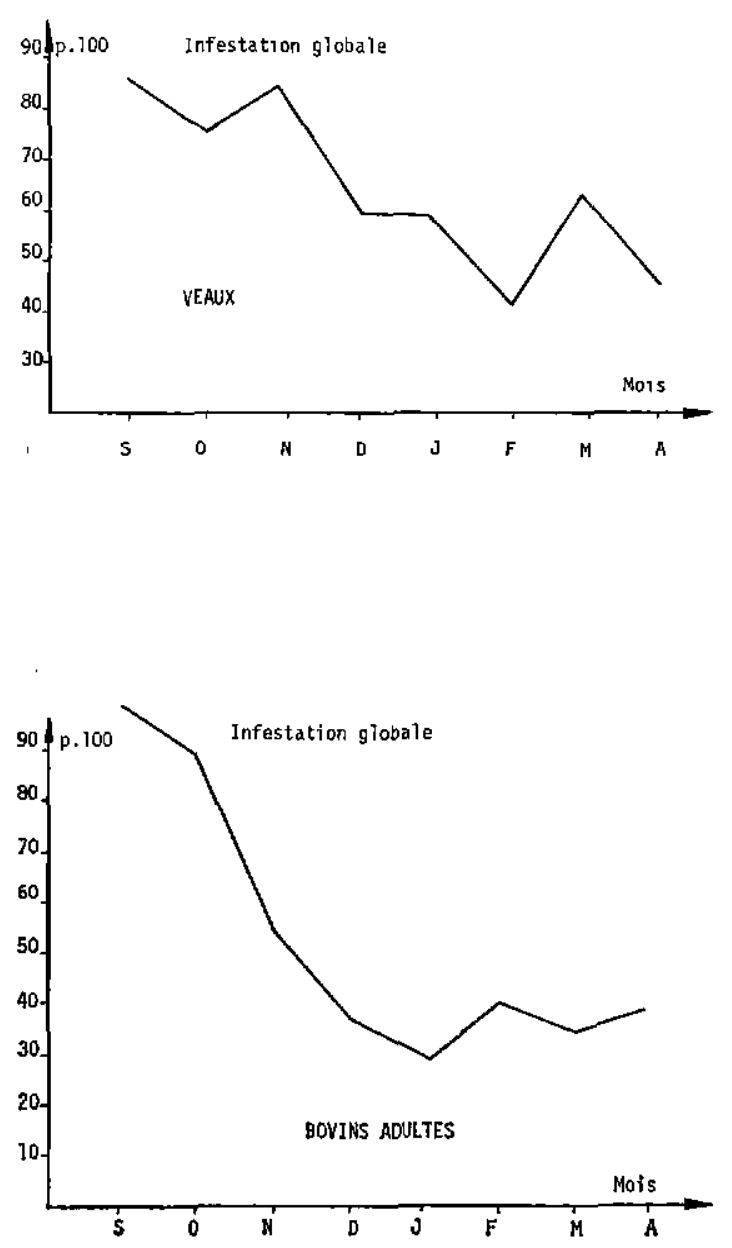

zurni chez les bovins, au genre Eimeria ninakohlyakimovae chez les ovins-caprins.

Les mesures quantitatives des divers éléments parasitaires montrent que les animaux sont toujours en dessous des seuils qui amènent des parasitoses maladies.

Les strongles dominants des Asins sont, par ordre décroissant d'importance : Triodontophorus, Strongylus, Trichonema, Trichostrongylus. Les numérations observées de janvier à avril correspondent à une extériorisation du parasitisme par des signes cliniques.

Les examens de viscères, pratiqués sur 30 bovins de 3 à 4 ans montrent que 77 p. 100 des animaux présentent des nodules dus aux larves d'Oesophagostomum; le nombre de nodule par animal est faible.
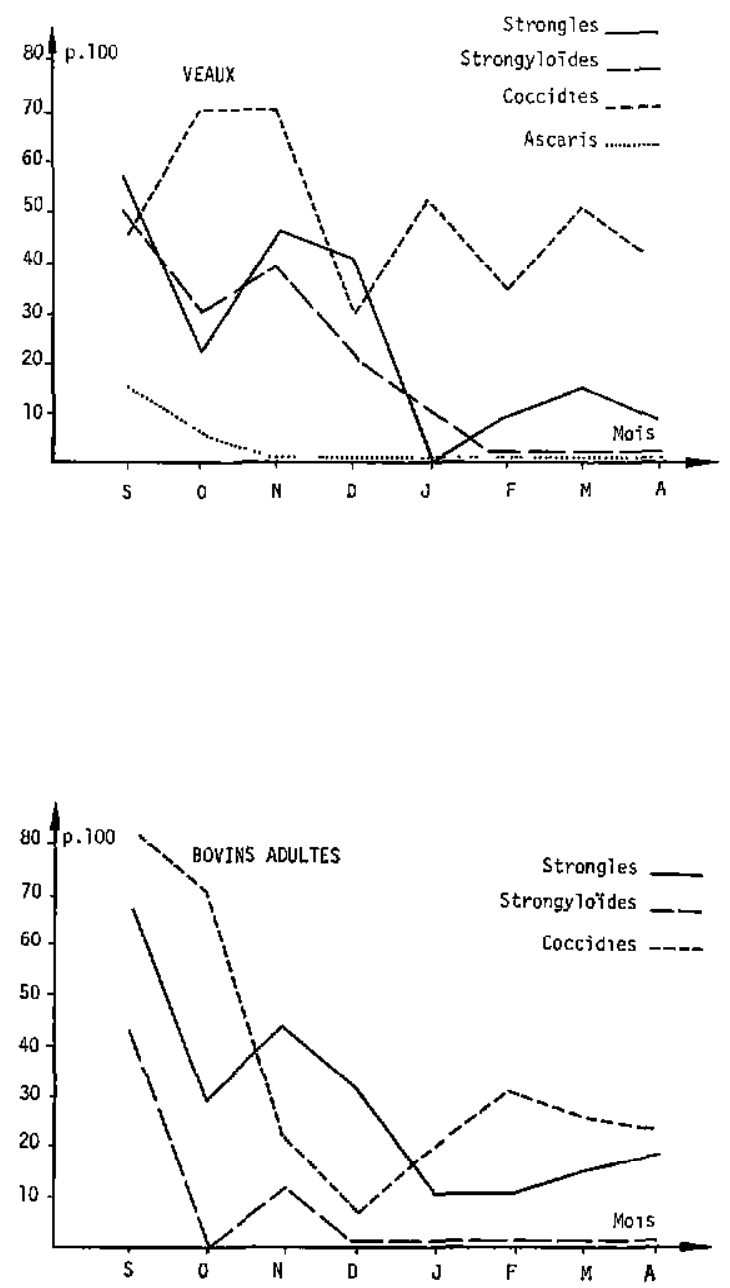

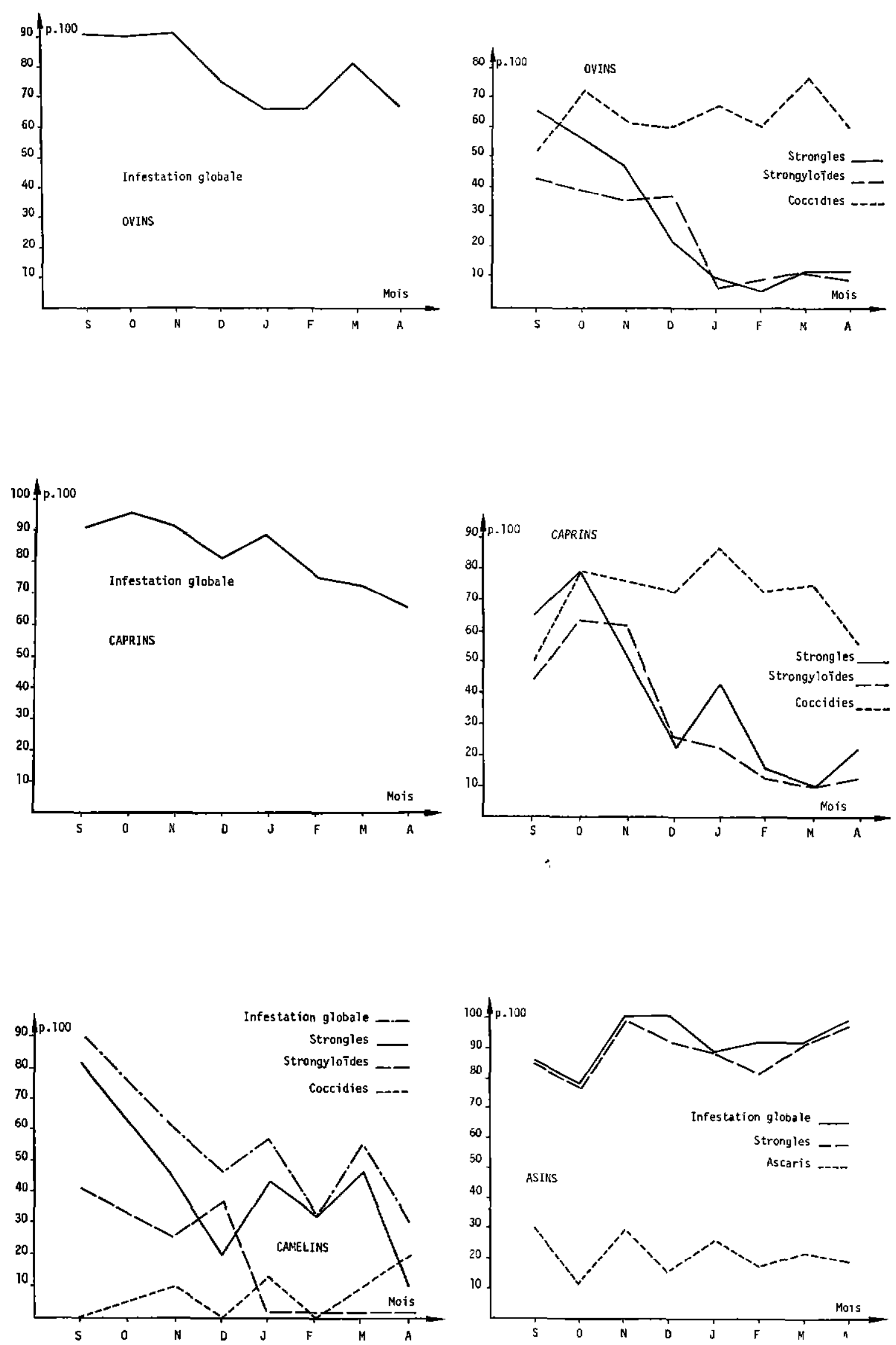


\section{EXPÉRIMENTATIONS}

A. Essai d'appréciation du gain de poids obtenu après traitement anti-parasitaire de masse sur les petits ruminants

L'expérience s'est déroulée du 2 décembre 1974 au 27 janvier 1975 sur le troupeau du Centre de Formation et de Vulgarisation agricole de Kaedi. Ce troupeau d'ovins-caprins vit dans des conditions proches de celles des troupeaux locaux. La moitié des animaux a été traitée un an auparavant au tétramisole lévogyre.

a) État parasitaire du troupeau en début d'expérience

Vingt prélèvements de fèces, pris au hasard, sont effectués le 2 décembre. Le taux d'infestation global est de 60 p. 100 . La structure parasitaire est :

coccidies: 50 p. 100 des animaux. 510 ookystes/g ;

strongyloïdes : 40 p. 100 des animaux. 225 cufs/g ;

strongles : 30 p. 100 des animaux. $210 œ u f s / g$.

b) Formation des lots

Trois lots sont formés par tirage au sort des animaux; les animaux des divers lots subissent les traitements suivants :

Lot $\mathrm{I}$ : tétramisole/lévogyre $=15 \mathrm{mg} / \mathrm{kg}$ vif; vif ;

Lot II : amprolium : dose unique de $8 \mathrm{mg} / \mathrm{kg}$

Lot III : témoin.

c) Évolution pondérale des animaux

TABL. $N^{\circ} \mathrm{I}$-Evolution pondérale des animaux.

\begin{tabular}{|l|c|c|c|}
\hline & $\begin{array}{c}\text { Lot I } \\
\mathrm{n}=25\end{array}$ & $\begin{array}{c}\text { Lot II } \\
\mathrm{n}=25\end{array}$ & $\begin{array}{c}\text { Lot III } \\
\mathrm{n}=20\end{array}$ \\
\hline 2 décembre 1974 & 28,1 & 28,2 & 28,7 \\
\hline 16 décembre 1974 & 27,6 & 28,1 & 28,4 \\
\hline 31 décembre 1974 & 27,8 & 28,8 & 29,0 \\
\hline 27 janvier 1975 & 29,8 & 31,0 & 31,0 \\
\hline
\end{tabular}

d) Étude des résultats

- État parasitaire : il n’y a pas d'amélioration de l'état parasitaire chez les animaux traités au bout de 55 jours d'expérience.

- Gains de poids.

L'analyse de covariance des poids du début et de fin d'expérience $(F=1,1)$ ne permet pas de mettre en évidence de différence significative entre les lots. Il en est de même pour les souslots « jeunes ". L'examen des données de pesées intermédiaires amène les mêmes résultats.

\section{Conclusions}

Dans les conditions de l'expérience : début de saison sèche, animaux traités en contact permanent avec des animaux non traités :

- on ne remarque pas d'amélioration de l'état parasitaire des troupeaux traités ;

- aucun gain de poids supplémentaire chez les animaux traités n'a pu être mis en évidence, au bout de deux mois (par rapport aux témoins)

\section{B. Essai de traitement de la coccidiose caprine par le sulfaguanidine}

Une dose unique de trois grammes par animal est administrée aux adultes et pré-adultes.

Un animal dont on peut disposer facilement sert à guider les essais : les ookystes qui ont presque disparu au bout de 4 jours, ont repris leur niveau initial 12 jours après le traitement.

groupe de 3 chèvres:

nombre d'ookystes/g : 61000 avant traitement (moyenne) $\quad 30 \quad 4$ jours après

groupe de 7 chèvres : nombre d'ookystes/g : 6200 avant traitement (moyenne) 5003 jours après 100011 jours après

L'administration de sulfaguanidine amène une diminution nette de l'émission d'ookystes au bout de 3 à 4 jours. Le traitement est actif. L'amélioration est de courte durée si les animaux utilisent les mêmes aires de repos qu'avant d'être déparasités. 10 à 11 jours après le traitement le taux d'infestation reprend sa valeur antérieure, avec une dispersion individuelle plus faible.

\section{Essai de détermination du moment optimal pour la prise de prélèvement fécal lors de la recherche des ookystes de coccidie}

Dans les conditions de la pratique, les prélèvements peuvent s'effectuer aussi facilement le matin que le soir, les animaux étant au pâturage dans la journée. Nous avons cherché lequel des examens (matin à 8 heures, soir à 19 heures) était le plus efficace.

26 ovins caprins sont utilisés, en saison sèche chaude. Les valeurs moyennes obtenues sont: 
620 ookystes/g le matin, 910 ookystes le soir.

Le test de $t$ apparié après changement de variable met en évidence une différence significative.

Il semble donc préférable d'effectuer les prélèvements fécaux le soir lors de recherche d'ookystes.

\section{DISCUSSION}

\section{A. Importance et nature du parasitisme - moyens de lutte}

Deux groupes d'animaux domestiques se distinguent à l'examen des taux d'infestation globale. L'un présente des variations saisonnières faibles (Asins, Equins), l'autre des fluctuations importantes (Ruminants).

Le premier groupe est le seul où les mesures quantitatives permettent de soupçonner une parasitose-maladie; c'est le cas de la strongylose dès le mois de janvier. On notera que les chevaux sont moins parasités que les ânes, sans doute pour des raisons d'ordre alimentaire.

Dans le second groupe, le maximum d'infestation est constaté de septembre à décembre. L'infestation coccidienne ne varie pas significativement au cours de la période considérée, sauf pour les bovins adultes où elle est maximale en septembre-octobre. Les strongles sont en diminution nette dès décembre-janvier. Il en est de même pour les strongyloïdes chez les petits ruminants ; pour les bovins et les camelins, on note leur disparition à cette époque. Les jeunes bovins sous la mère ont des ascaris seulement en septembre-octobre. Les jeunes caprins présentent une structure parasitaire peu différente de celle des adultes; seule l'infestation coccidienne pourrait être plus importante.

La date des opérations de vermifugation ne semble pas être importante chez les asins. La période favorable pour de telles pratiques chez les ruminants est vraisemblablement septembreoctobre, pour la lutte contre les strongles et les strongyloïdes.

La présence de ces derniers impose de traiter tous les ruminants sans distinction. Étant donné la présence de Trichostrongylus, il semble sage d'y inclure aussi les ânes.
Enfin, il faut essayer de traiter le plus d'animaux possible dans une région donnée. En effet, les animaux drogués auront tendance à se réinfester assez vite s'ils sont en contact avec des animaux parasités. Les résultats relatifs aux traitements de masse anti-coccidiens, tels qu'ils sont réalisés dans la pratique, sont décevants ; des mesures d'hygiène (déplacement des aires de repos par exemple) sont indispensables.

\section{B. Intérêt économique d'une action anti-para- sitaire de masse}

La seule mesure objective à court terme est l'évaluation du gain de poids. C'est évidemment une simplification qui ne tient pas compte de phénomènes importants tels : réduction de la mortalité, naissance de jeunes plus gros, résistance accrue aux divers stress ou infections, etc.

Chez les ovins-caprins, ni le tétramisole, ni l'amprolium à dose unique n'ont provoqué de gains de poids, dans une expérience réalisée en décembre-janvier. Les animaux, étant donné la division en lots, étaient susceptibles de se réinfester. L'intérêt économique de tels traitements n'a pu être prouvé dans ces conditions. Les remarques des éleveurs qui ont utilisé des minéraux et des anthelminthiques vont dans le même sens : leur faveur va aux compléments minéraux.

\section{Conclusion}

La fréquence des infestations pasasitaires chez les animaux domestiques utilitaires dans la région de Kaedi est élevée. L'essentiel des parasitoses peut se regrouper dans la grille : strongles-strongyloïdes, coccidies. Seuls les asins ne présentent pas de variations saisonnières. Les traitements anti-coccidiens restent décevants dans les conditions de la pratique. Des traitements de masse peuvent se concevoir dans la lutte contre les strongles et les strongyloïdes. La période favorable semble être septembreoctobre. L'anthelminthique devrait avoir les caractéristiques suivantes : absence de toxicité pour les principales espèces domestiques, activité contre les ascaris, strongles, strongyloïdes. Les principales espèces devraient être traitées, en nombre important dans chaque localisation géographique. L'intérêt économique de telles mesures demande à être évalué de façon précise. 


\section{SUMMARY}

Note about parasitism caused by nematodes and coccidia in domestic species in Kaedi area (Mauritania).

A parasitological survey was made from September 1974 to April 1975 in Kaedi area. The frame Strongle-Strongyloides-Coccidia for Ruminants and Strongle-Ascaris for donkeys is covering the essential of parasitism.

Infestation rates are high and are bound to season variations among Ruminants. Mass-treatments in September-October could be interesting on a sanitary level, for Strongle and Strongyloides if they are made on a maximum number of animals in a definite area, among the most important domestic species. On an economical level, such treatment should be backed by more experiments, which could take all variations into account.

\section{RESUMEN}

Nota sobre el parasitismo causado por los nemátodos y las coccidias en las especies domésticas en la region de Kaedi (Mauritania)

Se realizó una encuesta parasitaria sobre las principales especies domesticas de la region de Kaedi, de septiembre de 1974 a abril de 1975 . Un conjunto Estrong1los-Estronglloidos-Coccidias en los rumiantes y Estrongilos-Ascaris en los asnos representa el esencial del parasitismo.

Las tasas de infestación son elevadas y pueden variar según las estaciones en los rumiantes.

Los tratamientos intensivos en septiembre y octubre pueden tener un interes al punto de vista sanitario para los Estrongilos y los Estrongiloides si se efectuan en un maximo de animales en una region dada y en las principales especies domésticas. El interes económico de tales medidas necesita experimentaciones complementarias que tendrian en cuenta todos los factores. 